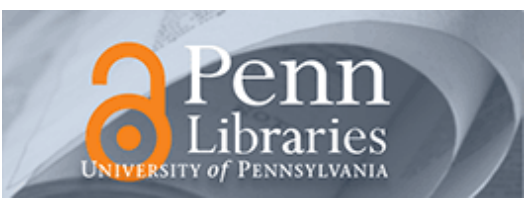

University of Pennsylvania

ScholarlyCommons

December 2000

\title{
Aggressive maneuvering of a thrust vectored flying wing: A receding horizon approach
}

\author{
John Hauser \\ University of Colorado \\ Ali Jadbabaie \\ University of Pennsylvania, jadbabai@seas.upenn.edu
}

Follow this and additional works at: https://repository.upenn.edu/ese_papers

\begin{abstract}
Recommended Citation
John Hauser and Ali Jadbabaie, "Aggressive maneuvering of a thrust vectored flying wing: A receding horizon approach", . December 2000.
\end{abstract}

Copyright 2000 IEEE. Reprinted from Proceedings of the 39th IEEE Conference on Decision and Control 2000,

Volume 4, pages 3582-3587.

Publisher URL: http://dx.doi.org/10.1109/CDC.2000.912261

This material is posted here with permission of the IEEE. Such permission of the IEEE does not in any way imply IEEE endorsement of any of the University of Pennsylvania's products or services. Internal or personal use of this material is permitted. However, permission to reprint/republish this material for advertising or promotional purposes or for creating new collective works for resale or redistribution must be obtained from the IEEE by writing to pubs-permissions@ieee.org. By choosing to view this document, you agree to all provisions of the copyright laws protecting it.

NOTE: At the time of publication, author Ali Jadbabaie was affiliated with the California Institute of Technology. Currently (March 2005), he is a faculty member in the Department of Electrical and Systems Engineering at the University of Pennsylvania.

This paper is posted at ScholarlyCommons. https://repository.upenn.edu/ese_papers/91

For more information, please contact repository@pobox.upenn.edu. 


\title{
Aggressive maneuvering of a thrust vectored flying wing: A receding horizon approach
}

\author{
Abstract \\ This paper deals with the control of a thrust vectored flying wing known as the ducted fan, developed at \\ California Institute of Technology. The experiment was developed to serve as a testbed for nonlinear \\ control design. In an earlier paper, the authors reported simulation results based on a simplified (no \\ aerodynamics involved) planar model of the ducted fan around hover position. In this paper we report on \\ the modeling and simulation of the ducted fan in forward flight, where aerodynamic forces and moments \\ can no longer be ignored. A receding horizon scheme is developed to generate trajectories for the forward \\ flight model. Using a more simplified version of the model, some aggressive trajectories are generated. \\ These trajectories are then used as a reference in the receding horizon scheme, and morphed into the \\ trajectories of the full model. Simulation results depict the capabilities of the ducted fan as well as this \\ methodology in performing aggressive maneuvers.
}

\section{Keywords}

aerospace control, military aircraft, nonlinear control systems, remotely operated vehicles, aerodynamic forces, aggressive maneuvering, ducted fan, forward flight, hover position, nonlinear control design, receding horizon approach, thrust vectored flying wing, thrust vectored aircraft, receding horizon control

\section{Comments}

Copyright 2000 IEEE. Reprinted from Proceedings of the 39th IEEE Conference on Decision and Control 2000, Volume 4, pages 3582-3587.

Publisher URL: http://dx.doi.org/10.1109/CDC.2000.912261

This material is posted here with permission of the IEEE. Such permission of the IEEE does not in any way imply IEEE endorsement of any of the University of Pennsylvania's products or services. Internal or personal use of this material is permitted. However, permission to reprint/republish this material for advertising or promotional purposes or for creating new collective works for resale or redistribution must be obtained from the IEEE by writing to pubs-permissions@ieee.org. By choosing to view this document, you agree to all provisions of the copyright laws protecting it.

NOTE: At the time of publication, author Ali Jadbabaie was affiliated with the California Institute of Technology. Currently (March 2005), he is a faculty member in the Department of Electrical and Systems Engineering at the University of Pennsylvania. 


\title{
Aggressive maneuvering of a thrust vectored flying wing: A receding horizon approach ${ }^{1}$
}

\author{
John Hauser ${ }^{2}$ and Ali Jadbabaie ${ }^{3}$ \\ \{hauser,alij\}@cds.caltech.edu
}

\begin{abstract}
This paper deals with the control of a thrust vectored flying wing known as the ducted fan, developed at California Institute of Technology. The experiment was developed to serve as a testbed for nonlinear control design. In an earlier paper, the . authors reported simulation results based on a simplified (no aerodynamics involved) planar model of the ducted fan around hover position. In this paper we report on the modeling and simulation of the ducted fan in forward flight, where aerodynamic forces and moments can no longer be ignored. A receding horizon scheme is developed to generate trajectories for the forward flight model. Using a more simplified version of the model, some aggressive trajectories are generated. These trajectories are then used as a reference in the receding horizon scheme, and morphed into the trajectories of the full model. Simulation results depict the capabilities of the ducted fan as well as this methodology in performing aggressive maneuvers. Keywords: ducted fan, thrust vectored aircraft,receding horizon control,nonlinear control design.
\end{abstract}

\section{Introduction}

There has recently been a lot of interest both in academia and industry to develop control methodologies for Unmanned Combat Aerial Vehicles (UCAVs). It is suggested that these vehicles will dominate the future of Aerial warfare. The fact that these vehicles are required to be autonomous and be able to perform aggressive maneuvers has

\footnotetext{
${ }^{1}$ Research supported in part by AFOSR and DARPA.

${ }^{2}$ Electrical and Computer Engineering, University of Colorado, Boulder, CO 80309-0425.

${ }^{3}$ Control and Dynamical Systems, Mail Code 107-81, California Institute of Technology, Pasadena, CA 91125.
}

required use of nonlinear control methodologies. Among all possible approaches, optimization based controllers have been a major candidate for controller design. Due to availability of faster and cheaper computing power, it is understood that a successful strategy should utilize the vast amount of computing power which is available now and is expected to grow even more in the future. Among current control strategies, receding horizon control seems to be a perfect candidate for these demanding control problems. Despite its success in process control industry, receding horizon control has not been employed in the aerospace industry.

Recently, the authors have proposed receding horizon schemes that are stabilizing and do not require imposing stability constraints in the optimization [3]. These results were applied to a simplified model of an experimental testbed developed at the California Institute of Technology. The experimental setup is known as the ducted fan $[2,5]$. The purpose of this paper is to apply the receding horizon control methodologies to the more sophisticated problem of maneuvering, where aerodynamic forces can no longer be ignored and the dynamics are highly nonlinear. The main challenge, as in many other flight control problem, is to generate trajectories in the presence of control constraints. Since the full model is not differentially flat, a more simplified model is used to generate a trajectory. Of course, this will not be a trajectory of the full model. However, using receding horizon schemes, one can morph the trajectory of the simplified model into the trajectory of the full model. Simulation results indicate that it is possible to perform very aggressive maneuvers using this approach.

This paper is organized as follows: Section 2 describes the aerodynamic modeling of the ducted fan as a thrust vectored flying wing. The reced- 
ing horizon morphing technique is described in section 3. Several aggressive maneuvers are generated in section 4. Finally, conclusions are presented in section 5 .

\section{Modeling of the Caltech Ducted Fan}

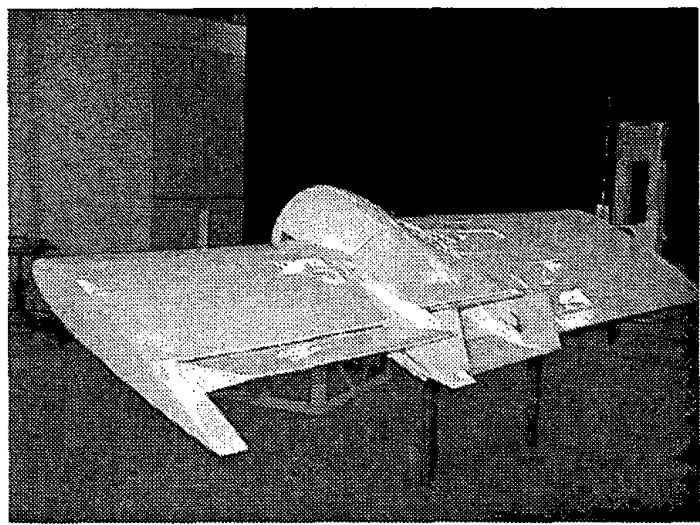

Figure 1: New Caltech Ducted Fan flight vehicle-July 99.

The Caltech Ducted Fan flight vehicle has been completely redesigned and rebuilt (July 1999) including new wings and better thrust vectoring to provide enhanced maneuvering capabilities. Initial flight tests and system modeling efforts confirm the desired improvements. This new flight vehicle provides an excellent experimental platform for development and testing of high performance maneuvering techniques for UCAVs.

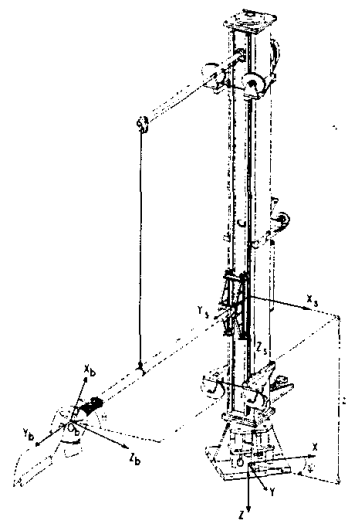

Figure 2: The Caltech ducted fan [5].

A significant amount of manual flying of the ducted fan has been accomplished to help understand

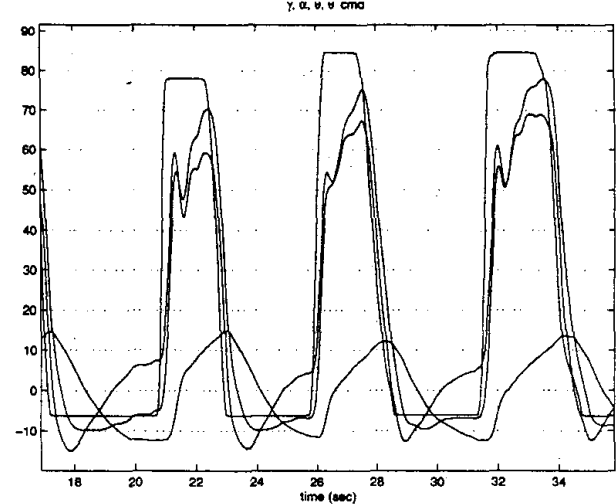

Figure 3: High AOA maneuvering of the Ducted Fan.

the flight characteristics of this unique flight vehicle. Through much practice and a number of minor (and major, yet survivable) crashes, we have gained courage and begun to expand our knowledge of the aggressive capabilities of the system. The ducted fan has been successfully flown through manual flip and turn around maneuvers. (These maneuvers are very difficult and manual attempts usually result in crashes!) Figure 3 shows flight data from some manual high angle of attack maneuvers. Note that we have been able to achieve an angle of attack of close to 70 degrees during dive recovery with flight path variations from -12 degrees up to 15 degrees.

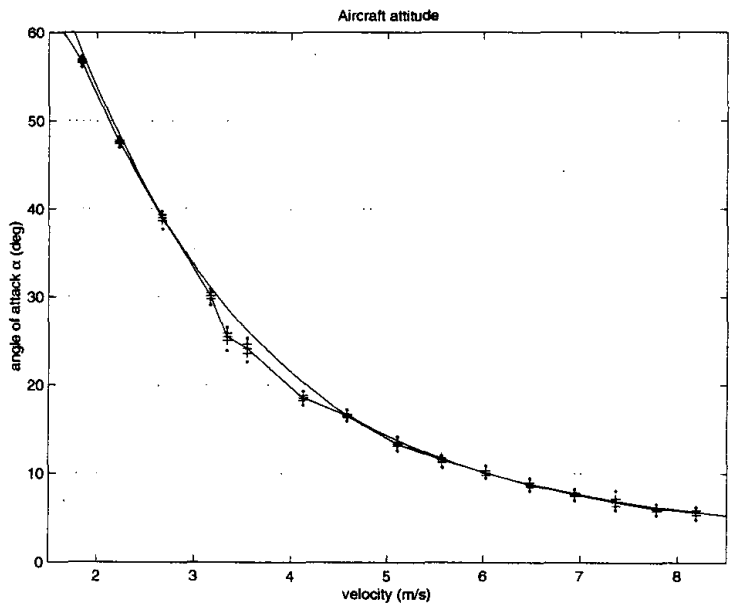

Figure 4: Experimental and model equilibrium manifolds. Angle of attack vs. velocity

We have initiated an activity to build up steady aerodynamic models using essentially steady state flight data. Obtaining steady flight data has 

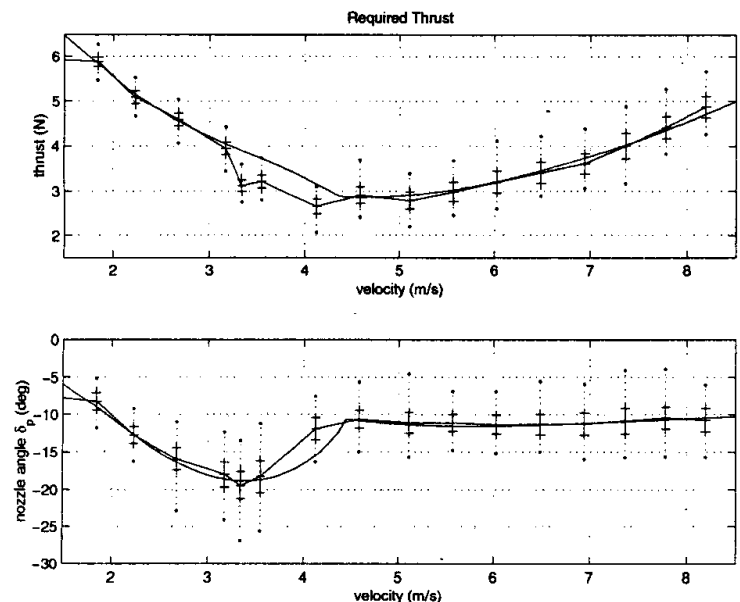

Figure 5: Experimental and model equilibrium manifolds. Required thrust vs. velocity

proved to be more challenging than expected due to a periodic drag force resulting from the interaction of a wing tip vortex with the walls, etc., in the vehicles lab. Nevertheless, early results look quite promising, especially for the purpose of obtaining a model with appropriate characteristics. Figures 4 and 5 show the experimental equilibrium manifold obtained by compiling many (steady) flight conditions. For each flight condition, the average, standard deviation, and range of values (min and max) are depicted.

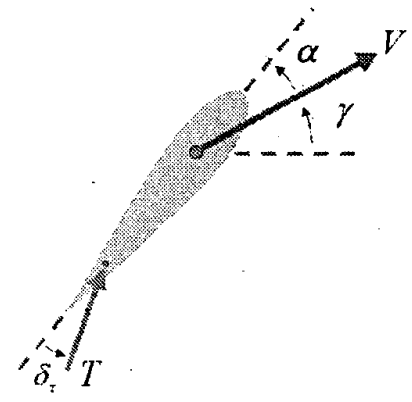

Figure 6: Flying wing with vectored thrust.

We have chosen to model the ducted fan as a flying wing with vectored thrust as depicted in Figure 6 . The effect of the newly designed thrust vectoring nozzle is modeled as a (vectored) force applied at a fixed position on the flight vehicle. The parameters for this model were estimated using static force/moment measurements. Using the experimental equilibrium manifold together with estimates of the thrust and thrust angle (obtained from the thrust model), we were able to scare out a plausible aerodynamic model, depicted in Figure 7 and 8 . Note the kink in the drag coefficient curve indicating that stall occurs between 16 and 20 degrees angle of attack. It is interesting to see that the lift curve does not exhibit the usual drop (or droop) after the wing stalls. We suspect that, at these angles of attack, the fuselage housing the fan begins to act like a lifting body, sustaining the lift force as the wing stalls.

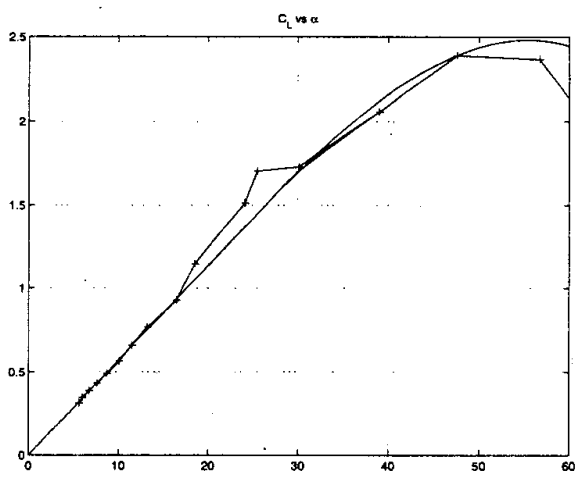

Figure 7: Experimental and model lift curves. The angle of attack values are in degrees.

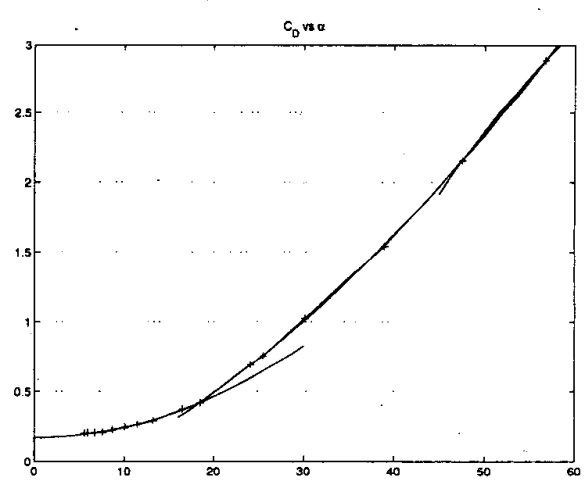

Figure 8: Experimental and model drag curves. The angle of attack values are in degrees.

Much more noticeable is the change in the pitching moment that occurs at stall. Although the moment curve is not shown, the effect is easily discernible in the equilibrium manifold nozzle angle curve of Figure 5. We were very pleasantly surprised to find that the model developed by fitting the lift, drag, and moment curves (see solid lines in Figures 7 and 8) resulted in a rather nice fit to the experimental equilibrium manifold in Figures 4 and 5 . We have begun to use this more sophisti- 
cated ducted fan model in our maneuver planning and execution studies, described below.

The equations of motion for the vectored thrust flying wing of Figure 6 are

$$
\begin{aligned}
m \dot{V} & =-D(V, \alpha)-W \sin \gamma+T \cos \left(\alpha+\delta_{\tau}\right) \\
m V \dot{\gamma} & =L(V, \alpha)-W \cos \gamma+T \sin \left(\alpha+\delta_{\tau}\right) \\
J \dot{q} & =M(V, \alpha)-T l_{\tau} \sin \delta_{\tau} \\
\dot{\theta} & =q
\end{aligned}
$$

where $\gamma=\theta-\alpha, L(V, \alpha)=\frac{1}{2} \rho V^{2} S C_{L}(\alpha)$, and $D(V, \alpha)=\frac{1}{2} \rho V^{2} S C_{D}(\alpha)$. It will be natural to take as state and control variables $x=(V, \gamma, q, \alpha)$ and $u=\left(T, \delta_{\tau}\right)$. The physical parameters for this model are $m=12.5 \mathrm{~kg}, g=0.6 \mathrm{~m} / \mathrm{s}^{2}, S=0.6 \mathrm{~m}^{2}$, $\rho=1.2 \mathrm{~kg} / \mathrm{m}^{3}, l_{\tau}=0.31 \mathrm{~m}, J=0.24 \mathrm{~kg} \mathrm{~m}^{2}$. Locally (i.e., up to stall), the lift and drag curves are given by

$$
\begin{array}{ll}
C_{L}(\alpha)=c_{l_{\alpha}} \alpha & =3.256 \alpha \\
C_{D}(\alpha)=c_{d_{0}}+c_{d_{\alpha}} \alpha^{2} & =0.1716+2.395 \alpha^{2}
\end{array}
$$

for $\alpha$ in radians. Experimental equilibrium manifold data provides information out to about 55 degrees angle of attack. Using symmetry considerations together with rather wild guesses, we have postulated an aerodynamic model that covers the full range through 180 degrees $\alpha$. Further verification/identification in that arena must be done using data from aggressive maneuvers. We also point out that we have made no attempt to model dynamic effects that depend on $\dot{x}$.

Roughly speaking, the thrust can range from 0 to $13.5 \mathrm{~N}$ and can be vectored a little more than 25 degrees $\left(\left|\delta_{\tau}\right| \leq 0.45\right)$. In terms of the input variables, $\delta_{p}$ (for commanded paddle angle) and $V_{m}$ (for motor voltage input), the thrust vector model is given by $T=46.5 V_{m}-5$ and $\delta_{\tau}=0.684 \delta_{p}$. This is a highly simplified, static model, making no attempts to model, for example, changes in thrust due to variations in speed and nozzle geometry.

Overall, we believe that this is a plausible model for the Caltech Ducted Fan, possessing many of the important characteristics of the real system. Much work remains in refining the model structure and parameters and in understanding the nature of uncertainties, both model and disturbance.

\section{Flight maneuvering and trajectory morphing}

As we have seen above, the Caltech Ducted Fan, modeled as a vectored thrust flying wing, is a highly nonlinear system with unique capabilities. From experience (and simple experiments), we know that operation near steady flight conditions can be easily achieved. Moreover, it is not too difficult to compute the associated equilibrium state and control values (e.g., as used in modeling above). It is much more difficult matter to specify feasible non equilibrium trajectories, especially aggressive maneuvers that push the limits of performance.

The use of vectored thrust allows us to operate the ducted fan beyond the friendly linear aerodynamic regime, providing unique opportunities. For example, during a dive recovery, one may sacrifice a certain amount of energy (while creating high drag) for the sake of improved maneuverability (higher lift plus thrust vectoring). Indeed, at the ' 99 Paris Air show, the pilot of the Sukhoi SU-30MKI came very close to recovering his craft using thrust vectoring (though it was the enhanced maneuverability that got him into trouble in the first place!). Another example is the use of high drag, high $\alpha$ maneuvering to affect a rapid turnaround (esp. for the ducted fan).

How may we approach the specification of high performance, or aggressive, maneuvers? Although models of the sort described above are nearly differentially flat, we suspect that the presence of aerodynamic forces and moments breaks the necessary symmetries. Moreover, physical considerations, such as the fact that only positive thrust can produced (so that we will speed up if we go down a steep enough hill!), limit the immediate usefulness of such information.

One approach is to use the idea of trajectory morphing [1] to parameterize the trajectories of a complicated system by those of a simpler system. Using a homotopy connecting the simple system to the complicated one, one may morph simple trajectories to those of the complicated system. One thus seeks a simple system for which trajectory exploration and specification is tractable and that is sufficiently rich to capture the essential dynamic 
coupling of the target system.

In the case of the vectored thrust flying wing, one may obtain a simplified model by removing from consideration the (internal) pitch dynamics and then using the angle of attack $\alpha$ as a pseudocontrol together with thrust. This results in a system with two states and two controls:

$$
\begin{aligned}
m \dot{V} & =-D(V, \alpha)-W \sin \gamma+T \cos (\alpha) \\
m V \dot{\gamma} & =L(V, \alpha)-W \cos \gamma+T \sin (\alpha)
\end{aligned}
$$

where, $V$, and $\gamma$ are the states, and $T$ and $\alpha$ are the controls. Given a trajectory $\left(V_{d}(\cdot), \gamma_{d}(\cdot), T_{d}(\cdot), \alpha_{d}(\cdot)\right)$ of the system $(2)$, we build up a desired trajectory for the full system (1) by defining $q_{d}(\cdot)$ as the (approximate, if necessary) derivative of $\left(\gamma_{d}+\alpha_{d}\right)(\cdot)$ and choosing $\delta_{\tau, d}$ so that

$$
J \dot{q}_{d}=M\left(V_{d}, \alpha_{d}\right)-T_{d} l_{\tau} \delta_{\tau, d}
$$

if $\delta_{\tau, d}(\cdot)$ is sufficiently small (keeping the sin otherwise). The desired trajectory is then defined to be $x_{d}(\cdot)=\left(V_{d}, \gamma_{d}, q_{d}, \alpha_{d}\right)(\cdot)$ and $u_{d}(\cdot)=\left(T_{d}, \delta_{\tau, d}\right)(\cdot)$.

To find a similar trajectory of the complicated system (1), we can solve a least squares problem of the form

$$
\begin{array}{ccc}
\operatorname{minimize} & \frac{1}{2} \int_{t_{0}}^{t_{f}}\left\|\left(x(\tau)-x_{d}(\tau), u(\tau)-u_{d}(\tau)\right)\right\|^{2} d \tau \\
\text { subject to } & \dot{x}(t)=f(x(t), u(t)), \quad t \in\left[t_{0}, t_{f}\right] \\
& x\left(t_{0}\right)=x_{d}\left(t_{0}\right)
\end{array}
$$

where $f$ describes the dynamics of the complicated system and the integrand may be a weighted square. Also, we might include a terminal cost (for some very good reasons). Morphing makes use of the fact that we know that $\left(x_{d}, u_{d}\right)(\cdot)$ satisfies the equations of motion for the simplified system. Thus, after a suitable augmentation of the simplified state and controls, we may define a homotopy connecting the simple system $f_{0}$ and the complicated system $f$, e.g., $f_{\lambda}=(1-\lambda) f_{0}+\lambda f$. We know the solution of the above optimization problem when $f=f_{\lambda}$, it is simply $\left(x_{d}, u_{d}\right)$. If $f$ and $f_{0}$ are well chosen (e.g., by a clever engineer), then $\lambda$ can be continued from 0 to 1 resulting in a trajectory of the complicated system that resembles the prototype trajectory of the simple system.

Of course, although the continuation may be useful, there are many cases where one may simple attack the optimization directly to obtain the complicated trajectory nearest the prototype (in the chosen $\mathcal{L}_{2}$ sense). Specifically, if the simplified system captures the essential dynamics and features of the more complicated model, one can also use a receding horizon scheme to morph the trajectories of the simplified model to those of the more complicated one, instead of using the above mentioned homotopy argument. Due to the fact that the receding horizon approach results in a sampled data feedback, a stabilizing controller around the trajectory is also generated, removing the need for a tracking controller.

How may we ensure the stability of the receding horizon scheme? We know from [3] that stability is guaranteed when a suitable CLF is used as terminal cost, when the goal is to regulate the system around the trivial trajectory, i.e., the equilibrium point. Since we are generating non-equilibrium trajectories, we would either need a time varying CLF derived by linearizing the dynamics along the generated trajectory, or use a long horizon length. In this case, since the trajectory is not known $a$ priori, a suitable CLF is by no means easy to find. It can be shown [4] that there always exist a $f$ nite horizon length for which the receding horizon scheme is stabilizing without a terminal cost or constraint. We will use this result to ensure stability of the morphing scheme.

\section{Aggressive Maneuvers}

In this section we present a few of the maneuvers obtained by implementing the morphing technique in a receding horizon fashion on maneuvers developed using the simplified model.

To get an idea of the maneuverability of the ducted fan, we first push the flying wing through a number of periodic climb/dive maneuvers similar to what we have flown of the real ducted fan. The period of the maneuvers was chosen to be 5 seconds. Figures 9 and 10 show the nature of these maneuvers. We see that the simplified system actually does a decent job of specifying approximate trajectories. A standard acrobatic maneuver for reversing direction is made up of a half loop, better known as an Immelman. For the ducted fan (in up and away flight sans floor and ceiling), the idea is to make the flight path angle $\gamma$ go from 0 degrees to 180 degrees. The piloted maneuver would 

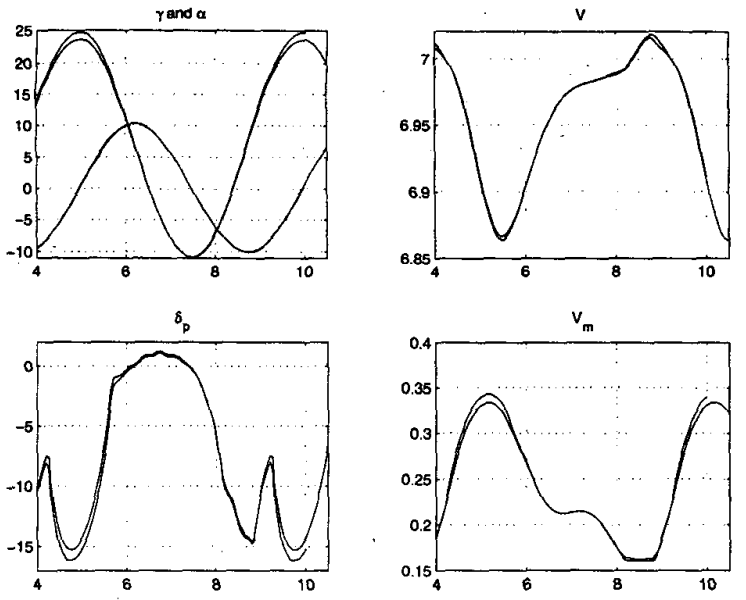

Figure 9: Periodic diving and climbing maneuver with approximately 20 degrees flight path angle $\gamma$ change. Note that the angle of attack $\alpha$ approaches 25 degrees.

be completed with a 180 degree roll but we have no roll axis with the ducted fan and, moreover, the ducted fan is quite happy flying inverted since the system is more or less symmetric. Figure 11 shows such a maneuver. Once again, the simplified system works quite well. In this figure, we have actually plotted the entire set of one second optimal trajectories (with artifacts between them).

\section{Conclusion}

The purpose of this paper was to report on the latest modeling and simulation results on an experimental thrust vectored flying wing developed at Caltech. It was shown that by solving a trajectory generation problem for a simplified model of the system and using that as a reference trajectory in a receding horizon framework, some aggressive maneuvers can be performed. A detailed description of the modeling efforts as well as capabilities of the experimental set up were discussed. The next step would be to apply this method to the actual experiment.

\section{References}

[1] J. Hauser and D. G. Meyer. Trajectory morphing for. nonlinear systems. In American Control Conference, 1998.

[2] A. Jadbabaie, J. Yu, and J. Hauser. Receding horizon control of the Caltech ducted fan: A control Lyapunov
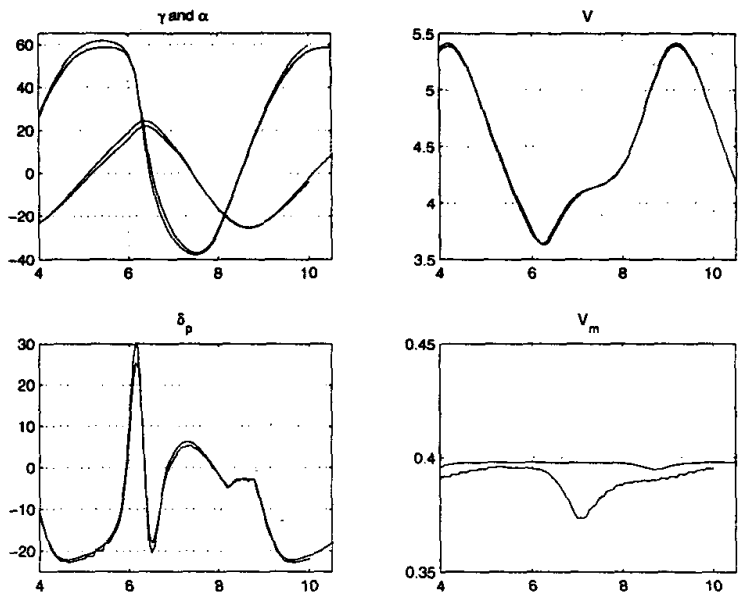

Figure 10: Periodic diving and climbing maneuver with approximately 50 degrees flight path angle $\gamma$ change. Note that the angle of attack $\alpha$ goes to higher than 60 degrees. Also, we see that the thrust is almost continuously at the maximum.

function approach. In IEEE Conference on Control Applications, 1999.

[3] A. Jadbabaie, J. Yu, and J. Hauser. Unconstrained receding horizon control of nonlinear systems. In $I E E E$ Conference on Decision and Control, 1999.

[4] A. Jadbabaies. Receding horizon control of nonlinear systems: $A$ control Lyapunov function approach. PhD thesis, under preparation, California Institute of Technology, Pasadena, CA, 2000.

[5] M. Milam and R. M. Murray. A testbed for nonlinear flight control techniques: The Caltech Ducted Fan. In IEEE Conference on Control Applications, Big Island, Hawaii, 1999.
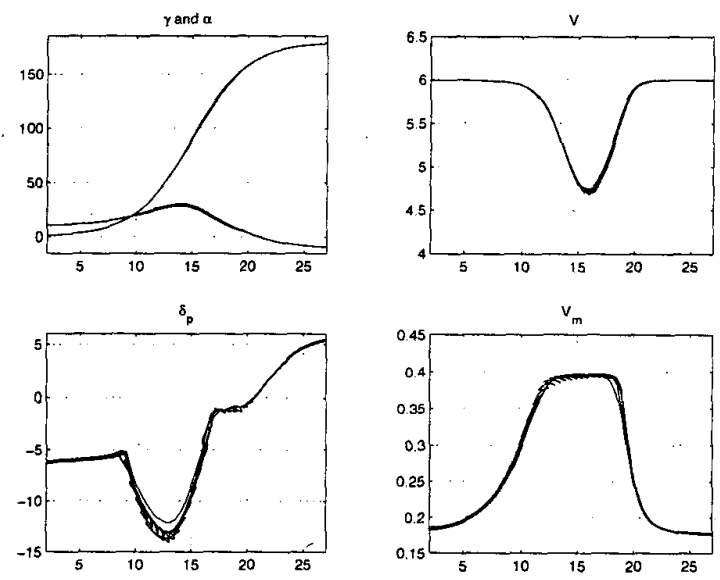

Figure 11: Immelman maneuver for reversing direction. Note the radical change in $\delta_{p}$ as stall is encouritered. 